\title{
Phylogenomic characterisation of a novel corynebacterial species pathogenic to animals
}

\author{
Jens Möller • Luca Musella • Vyacheslav Melnikov • Walter Geißdörfer • \\ Andreas Burkovski • Vartul Sangal (i)
}

Received: 12 March 2020/Accepted: 26 May 2020/Published online: 4 June 2020

(C) The Author(s) 2020

\begin{abstract}
The genus Corynebacterium includes species of biotechnological, medical and veterinary importance. An atypical C. ulcerans strain, W25, was recently isolated from a case of necrotizing lymphadenitis in a wild boar. In this study, we have analysed the genome sequence of this strain and compared the phenotypic and virulence properties with other corynebacterial pathogens. Phylogenomic analyses revealed that strain W25 belongs to a novel species along with PO100/5 and KL1196. The latter strains were isolated from a pig and a roe deer,
\end{abstract}

Electronic supplementary material The online version of this article (https://doi.org/10.1007/s10482-020-01430-5) contains supplementary material, which is available to authorized users.

J. Möller · L. Musella · A. Burkovski

Professur für Mikrobiologie, Friedrich-Alexander-

Universität Erlangen-Nürnberg, Erlangen, Germany

V. Melnikov

Gabrichevsky Research Institute for Epidemiology and

Microbiology, Moscow, Russia

W. Geißdörfer

Institute of Clinical Microbiology, Immunology and Hygiene, Universitätsklinikum Erlangen, Friedrich-

Alexander-Universität Erlangen-Nürnberg, Erlangen, Germany

\section{Sangal ( $\square)$}

Faculty of Health and Life Sciences, Northumbria

University, Newcastle upon Tyne, UK

e-mail: vartul.sangal@northumbria.ac.uk respectively; hence, this species appears to be associated to animals. The isolate W25 is likely a nontoxigenic tox gene bearing strain and may have compromised abilities to adhere to pharyngeal and laryngeal epithelial cells due to potential loss of the gene functions in $s p a B C$ and $s p a D E F$ pilus gene clusters. A number of corynebacterial virulence genes are present including pld encoding phospholipase D. Therefore, this strain may be able to cause severe invasive infections in animals and zoonotic infections in humans.

Keywords Corynebacterium ulcerans · Diphtheria · Non-toxigenic $\cdot$ NTTB $\cdot$ Toxigenic $\cdot$ Virulence . Zoonotic

\section{Introduction}

Corynebacterium is a diverse genus that includes species of biotechnological, medical and veterinary importance (Bernard and Funke 2015). One of the corynebacterial species, Corynebacterium ulcerans, is an important zoonotic pathogen often acquired from canine pets and causes diphtheria-like infections in humans (Hacker et al. 2016; Mattos-Guaraldi et al. 2014). C. ulcerans has also been isolated from other animals including camels, cattle, cats, goats, ground squirrels, monkeys, pigs, otters, whales, etc. 
[Reviewed by Hacker et al. (2016)]. A human-tohuman transmission of $C$. ulcerans has also been reported (Konrad et al. 2015).

The key virulence factor among C. ulcerans strains is the tox gene (Sangal and Hoskisson 2014; Sangal et al. 2014; Subedi et al. 2018); which is borne by a corynephage (Sekizuka et al. 2012) or pathogenicity island (Meinel et al. 2014). However, non-toxigenic tox gene bearing (NTTB) C. ulcerans strains, where tox gene is a pseudogene, are also common (Dias et al. 2011; Eisenberg et al. 2014; Fuursted et al. 2015; Wagner et al. 2011; Zakikhany et al. 2014). Other known virulence-associated genes in $C$. ulcerans include pld (phospholipase D), nanH (neuraminidase $\mathrm{H}$ ), $c p 40$ (corynebacterial protease), vsp1 and vsp2 (venom serine protease) and rbp (Sangal et al. 2014; Sekizuka et al. 2012; Subedi et al. 2018; Trost et al. 2011). The $r b p$ gene encodes a ribosomal-binding protein that is similar to Shiga-like toxin and is only reported in C. ulcerans strain 809 (Subedi et al. 2018; Trost et al. 2011). Interestingly, an Rbp homolog was found in C. diphtheriae HCO4 (Weerasekera et al. 2019).

Two Spa gene clusters, $s p a D E F$ and $s p a B C$, have been reported among C. ulcerans strains (Subedi et al. 2018; Trost et al. 2011). Pilus gene clusters encode surface pili that play a key role in adhesion and invasion to the host cells (Broadway et al. 2013; Reardon-Robinson and Ton-That 2014). A variation in the numbers of pilus gene clusters and gain or loss of gene function was found to correlate with differences in the severity of infection by Corynebacterium diphtheriae, another important human pathogen closely related to $C$. ulcerans (Grosse-Kock et al. 2017; Ott et al. 2010; Sangal et al. 2015).

We have recently isolated an atypical $C$. ulcerans strain, W25, associated with necrotizing lymphadenitis in a wild boar and published the genome sequence (Busch et al. 2019). While the size of the genome is consistent with other $C$. ulcerans genomes, $\mathrm{G}+\mathrm{C}$ content of the W25 was approximately $1.0 \%$ higher than other C. ulcerans strains (Busch et al. 2019). It may reflect significant variations in the gene content and virulence properties of this strain than other $C$. ulcerans isolates. Therefore, we compared the phenotypic and virulence properties of this strain and performed a comparative genomic analysis against other $C$. ulcerans isolates.

\section{Materials and methods}

Bacterial strains and culture conditions

Eight corynebacterial strains were included for phenotypic characterisation (Table 1). The strains were cultured in Brain Heart Infusion (BHI) broth at $37^{\circ} \mathrm{C}$ and were incubated overnight in a shaking incubator.

Strain identification

Strain W25 was analysed by MALDI-TOF mass spectrometry as previously described (Alibi et al. 2015). Biochemical tests were performed for strain W25 using the standard approach (Efstratiou and George 1999). Antimicrobial susceptibility testing method was carried out on Müller-Hinton agar as described in detail on the EUCAST website (http:// www.eucast.org).

\section{Multiplex PCR}

For differentiation of Corynebacterium species, a multiplex colony PCR, based on five genes, $r p o B, 16 S$ $r R N A, p l d, d t x R$ and tox, was performed using the oligonucleotides listed in Table 2. For colony PCR, a loopful of freshly grown bacteria was resuspended in $500 \mu \mathrm{l}$ of sterile deionized water and boiled for $10 \mathrm{~min}$ at $95{ }^{\circ} \mathrm{C}$. The suspension was centrifuged at $13,000 \times \mathrm{g}$ for $1 \mathrm{~min}$ and $1 \mu \mathrm{l}$ of the supernatant was used as template. Multiplex PCR was carried out in a Primus 96 advanced thermocyler (Peqlab, Erlangen) using previously described conditions (Torres Lde et al. 2013). The amplicons were separated by electrophoresis on a $3 \%$ agarose gel.

\section{Detection of $C$. diphtheriae toxin production}

Elek test (reaction of immunoprecipitation) was performed as described in the Manual for Diphtheria Laboratory Diagnosis (Efstratiou and George 1999; Mazurova et al. 1998). Korinetoksagar (State Research Center for Microbiology and Biotechnology, Obolensk, Russia) with $15 \%$ fetal calf serum was used to grow the bacterial strains. Filter paper strips $(8.0 \times 1.3 \mathrm{~cm})$ were impregnated with $0.25 \mathrm{ml}(500$ IU in $1 \mathrm{ml}$ ) of purified diphtheria antitoxin (Microgen, Russia) and placed on the centre of the agar plates. Recommended control strains, toxigenic $C$. 
Table 1 Bacterial strains used in this study

\begin{tabular}{|c|c|c|}
\hline Strain & Description/source & References \\
\hline C. glutamicum ATCC 13032 & Soil (tox ${ }^{-}$) & Abe et al. (1967) \\
\hline C. diphtheriae ISS3319 & Human $\left(\operatorname{tox}^{-}\right)$ & Sangal et al. (2015) \\
\hline C. diphtheriae NCTC 10648 & Human $\left(\operatorname{tox}^{+}\right)$ & \\
\hline C. diphtheriae NCTC 10356 & Human nose (tox ${ }^{-}$) & \\
\hline C. ulcerans 809 & Human $\left(\operatorname{tox}^{-}\right)$ & Dias et al. (2010) \\
\hline C. ulcerans $\mathrm{BR}-\mathrm{AD} 22$ & $\operatorname{Dog}\left(\operatorname{tox}^{-}\right)$ & Mattos-Guaraldi et al. (2008) \\
\hline C. ulcerans $\mathrm{KL} 756$ & $\operatorname{Dog}\left(\operatorname{tox}^{+}\right)$ & Möller et al. (2019) \\
\hline C. ulcerans W25 & Wild boar $\left(\right.$ tox $\left.^{+}\right)$ & Busch et al. (2019) \\
\hline
\end{tabular}

Table 2 Oligonucleotides used in this study

\begin{tabular}{|c|c|c|}
\hline Name & Sequence $\left(5^{\prime} \rightarrow 3^{\prime}\right)$ & Description \\
\hline \multicolumn{3}{|c|}{ RNA hybridization } \\
\hline DIP2222-s & GTCTCACTGAACCGTTGATG & Forward primer for tox gene \\
\hline $\begin{array}{l}\text { DIP2222- } \\
\text { T7as }\end{array}$ & CCCGGGTAATACGACTCACTATAGGGCGCTATCGATAACTTGCGCAACG & Reverse primer for tox gene \\
\hline 16SrRNA-s & GCAGCCGCGGTAATACGTAG & $\begin{array}{l}\text { Forward primer for } 16 S r R N A \\
\text { gene }\end{array}$ \\
\hline 16SrRNA-as & GGGCCCTAATACGACTCACTATAGGGACATCT CACG ACAC GAGCTG & $\begin{array}{l}\text { Reverse primer for } 16 S \text { rRNA } \\
\text { gene }\end{array}$ \\
\hline \multicolumn{3}{|c|}{ Multiplex PCR } \\
\hline $\mathrm{C} 2700 \mathrm{~F}$ & CGTATGAACATCGGCCAGGT & $r р о B$ \\
\hline C3130 R & TCCATTTCGCCGAAGCGCTG & rров \\
\hline $16 \mathrm{~S} \mathrm{~F}$ & ACCGCACTTTAGTGTGTGTG & $16 S r R N A$ \\
\hline $16 \mathrm{~S} R$ & TCTCTACGCCGATCTTGTAT & $16 S r R N A$ \\
\hline pld F & ATAAGCGTAAGCAGGGAGCA & pld \\
\hline pld $\mathrm{R}$ & TCAGCGGTGATTGTCTTCC & pld \\
\hline $\mathrm{dtxR} 1 \mathrm{~F}$ & GGGACTACAACGCAACAAGAA & $d t x R$ \\
\hline dtxR $1 R$ & CAACGGTTTGGCTAACTGTA & $d t x R$ \\
\hline dipht $4 \mathrm{~F}$ & GAACAGGCGAAAGCGTTAAGC & tox \\
\hline dipht $4 \mathrm{R}$ & TGCCGTTTGATGAAATTCTTC & tox \\
\hline
\end{tabular}

diphtheriae NCTC 10648, non-toxigenic C. diphtheriae NCTC 10356, and test cultures were transferred to the plate at a distance of 6-7 $\mathrm{mm}$ from the strip edge. The Elek test was analysed after $24 \mathrm{~h}$ of incubation at $37^{\circ} \mathrm{C}$.

SDS-PAGE and Western blotting

Corynebacterium ulcerans strains were incubated at $37{ }^{\circ} \mathrm{C}$ in a shaking incubator in BHI broth (Oxoid, Wesel) and were grown to an $\mathrm{OD}_{600}$ of 0.4-0.6. For toxin production, 2-2'-bipyridyl was added at a final concentration of $0.5 \mathrm{mM}$ during exponential phase and bacterial strains were incubated for further $2 \mathrm{~h}$ under iron starvation conditions (Moreira et al. 2003). The cells were harvested by centrifugation. Protein extraction, separation of proteins by SDS gel electrophoresis and immuno-detection of diphtheria toxin with human serum were carried out as described previously (Möller et al. 2019). 
RNA isolation and hybridization

RNA isolation and hybridization was carried out as described previously (Ott et al. 2010). For hybridization of the digoxigenin-labelled RNA probes (prepared by PCR using the oligonucleotides listed in Table 2) and detection, alkaline phosphatase-conjugated anti-digoxigenin Fab fragments and CSPD [disodium 3-(4-methoxyspiro\{1,2-dioxetane-3, 2' -(5'chloro)tricyclo [3.3.1.13,7]decan $\} 4$-yl)phenyl phosphate] (Roche, Mannheim) were used. Chemiluminescence was detected using a ChemiDoc XRS + system (BioRad, Munich).

Genome sequences

A scaffold was generated from the draft assembly of strain W25 (Accession number: VFEM00000000) against the genome of C. ulcerans strain PO100/5 using MeDuSa web-server (Bosi et al. 2015). The genome sequences of 28 other C. ulcerans strains and type strains of closely related corynebacterial species, C. diphtheriae, Corynebacterium belfanti and Corynebacterium pseudotuberculosis were obtained from the GenBank (Supplementary Table 1).

Phylogenomic analyses

16S rRNA gene sequence (1509 bp in size) was extracted from the genome sequence of strain W25 using RNAmmer v1.2 (Lagesen et al. 2007). The reference 16S rRNA gene sequences of all corynebacterial strains were obtained from the GenBank. The nucleotide sequences were aligned using MUSCLE (Edgar 2004) and a phylogenetic tree was constructed from resulting sequence alignment (1155 bp in size after excluding sites with gaps) using IQtree with 100,000 ultra-fast bootstraps and 100,000 SH-aLRT tests (Nguyen et al. 2015). The tree was visualised using iTOL (Letunic and Bork 2016). Pairwise average nucleotide identities (ANI) were calculated among $C$. ulcerans genome sequences and the type strains of closely related corynebacterial species using FastANI (Jain et al. 2018). Digital DNA-DNA hybridisation $(\mathrm{dDDH})$ values were calculated using Genome-to-Genome Distance Calculator 2.1 (Auch et al. 2010a, b). The genome sequence of strain W25 was also analysed using the PathoBacTyper (Tsai, Liu and Soo 2017), TrueBac ${ }^{\mathrm{TM}}$ ID cloud system (Ha et al.
2019) and Type (Strain) Genome Server (MeierKolthoff and Göker 2019).

All genome sequences were annotated using Prokka v 1.12 (Seemann 2014) and compared using Roary v 3.12.0 with an identity cut-off of 70\% (Page et al. 2015; Tange 2011). A maximum-likelihood tree was calculated from the core genomic sequence alignment after removing the sites with missing data using IQ-Tree with 100,000 ultra-fast bootstraps and 100,000 SH-aLRT tests (Nguyen et al. 2015).

Identification of virulence genes

The known virulence genes from pathogenic corynebacteria including $C$. diphtheriae, C. pseudotuberculosis as well as C. ulcerans were searched into the genome of strain W25 using the protein BLASTsearches (Altschul et al. 1997; Camacho et al. 2009).

Identification of genes involved in starch metabolism

Glycoside hydrolases that are responsible for hydrolysis of amylose and amylopectin were identified from the KEGG pathway for starch and sucrose metabolism (https://www.genome.jp/kegg-bin/show

pathway?map00500) and were searched among the protein sequences of strains W25, PO100/5 and KL1196, C. ulcerans strains BR-AD22 and NCTC 12077, and C. pseudotuberculosis DSM 20689 obtained from the GenBank (Supplementary Table 1) using protein-protein PSI-BLAST algorithm (Altschul et al. 1997). A "KEGG-inferred" database was created with identified glycoside hydrolases sequences.

A two-step protein BLAST search strategy was applied to refine these results and to identify enzymes conserved among all corynebacterial strains. In the first search, proteins sequences from all six strains were searched in the "KEGG-inferred" database using BLASTP (Camacho et al. 2009). The query sequences with significant similarity to sequences in the database ( $\geq 90 \%$ coverage, $\geq 60 \%$ identity and e-value $\leq 1 \mathrm{e}-$ 165 ) were aligned using Clustal-Omega (Sievers et al. 2011). The hierarchical clustering in the multiple sequence alignment allowed distinguishing protein groups, which were used to record a presence or absence of enzymes among individual strains. In the step 2, the proteins from each hierarchical cluster in 
the sequence alignment was used as the query against the entire proteome of the six corynebacterial strains. This was an additional confirmation of the presence or absence of a given enzyme in particular strains.

\section{Results}

Identification and biochemical characteristics of strain W25

Strain W25 was initially identified by MALDI-TOF mass spectrometry as $C$. ulcerans with a score of 2.065. Multiplex PCR amplified fragments of $16 \mathrm{~S}$ rRNA, $r p o B$ and tox genes with a faint DNA band for pld gene for strain W25 (Fig. 1), a profile consistent with other $C$. ulcerans isolates as the primers were designed to amplify the fragments of rpoB and tox genes for $C$. diphtheriae, C. pseudotuberculosis and $C$. ulcerans, 16S rRNA for C. ulcerans and C. pseudotuberculosis, pld for C. pseudotuberculosis and $d t x R$ for $C$. diphtheriae strains.

Isolate W25 was found to produce $\mathrm{H}_{2} \mathrm{~S}$ on Tinsdale medium when stabbed into the surface and was ureasepositive (Table 3 ). The strain was positive for reverse
CAMP reaction, i.e., produced phospholipase D inhibiting $\beta$-haemolysis by Staphylococcus aureus on blood-agar plates. The strain was also positive for hydrolase activity and was able to utilise glucose as a carbon source (Table 3). W25 could not hydrolyse gelatine, reduce nitrate or ferment starch and was negative for toxin production according to the Elek test (Table 3). The strain was sensitive to all antibiotics tested (Supplementary Table 2). The extensive search for glycoside hydrolases revealed the absence of two enzymes 1,4-alpha-amylase and a type I pullulanase, in the strain W25. These enzymes are involved in starch hydrolysis and an absence of these enzymes explains inability of W25 strain to ferment starch (Table 4).

Phylogenomic characterisation

The maximum-likelihood tree from 16S rRNA gene also grouped strain $\mathrm{W} 25$ with $C$. ulcerans isolates (Fig. 2). 16S rRNA sequence of strain W25 showed $99.65 \%$ similarity with the $16 \mathrm{~S}$ rRNA gene in $C$. ulcerans strain CD361 and $99.31 \%$ similarity with the 16S rRNA gene of $C$. ulcerans strain NCTC 7910.

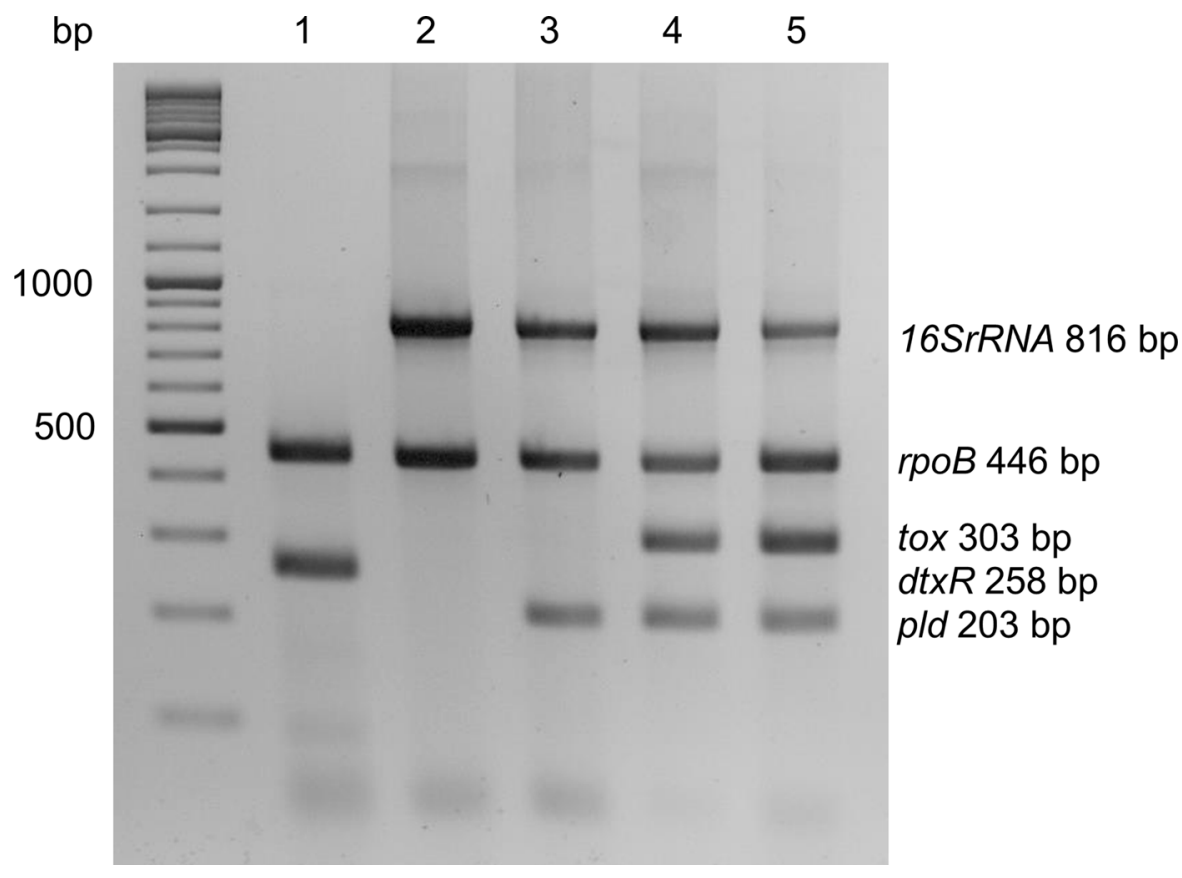

Fig. 1 Multiplex PCR for the identification of corynebacteria. Lane 1: C. diphtheriae ISS 3319 (tox ${ }^{-}$); 2: C. ulcerans 809 (tox ${ }^{-}$); $3:$ C. ulcerans BR-AD22 (tox ${ }^{-}$); 4: C. ulcerans $\mathrm{KL} 756$ (tox $\left.^{+}\right)$; 5: C. ulcerans W25 
Table 3 Biochemical characteristics of strain W25

\begin{tabular}{|c|c|c|c|c|c|c|c|c|c|}
\hline Strains & $\begin{array}{l}\text { Elek } \\
\text { test }\end{array}$ & $\mathrm{H}_{2} \mathrm{~S}$ & $\begin{array}{l}\text { Nitrate } \\
\text { reductase }\end{array}$ & Urease & $\begin{array}{l}\text { Reverse } \\
\text { camp }\end{array}$ & $\begin{array}{l}\text { DNAse } \\
\text { activity }\end{array}$ & $\begin{array}{l}\text { Gelantine } \\
\text { hydrolysis }\end{array}$ & Starch & Glucose \\
\hline C. diphtheriae ${ }^{*}$ & \pm & + & \pm & - & - & + & - & \pm & + \\
\hline C. ulcerans ${ }^{*}$ & \pm & + & - & + & + & + & \pm & + & + \\
\hline $\begin{array}{l}\text { C. } \\
\text { pseudotuberculosis }\end{array}$ & \pm & + & \pm & + & + & - & \pm & \pm & + \\
\hline Strain W25 & - & + & - & + & + & + & - & - & + \\
\hline
\end{tabular}

*Expected results taken from Bernard and Funke (2015), Dorella et al. (2006), and Mattos-Guaraldi et al. (2014)

Table 4 A presence and absence of two enzymes involved in starch metabolism

\begin{tabular}{|c|c|c|}
\hline Protein name & Strain & NCBI Protein ID \\
\hline \multirow[t]{6}{*}{ Pullulanase type I (Protein cluster: PCLA_2760914) } & C. ulcerans $\mathrm{BR}-\mathrm{AD} 22$ & AEG83474.1 \\
\hline & C. ulcerans NCTC 12077 & ESU58386.1 \\
\hline & W25 & Absent \\
\hline & PO100/5 & Absent \\
\hline & KL1196 & Absent \\
\hline & C. pseudotuberculosis DSM20689 & RKT29492.1 \\
\hline \multirow[t]{6}{*}{ 1,4-Alpha amylase (Protein cluster: PCLA_3428021) } & C. ulcerans $\mathrm{BR}-\mathrm{AD} 22$ & AEG82814.1 \\
\hline & C. ulcerans NCTC 12077 & ESU59224.1 \\
\hline & W25 & Absent \\
\hline & $\mathrm{PO} 100 / 5$ & Absent \\
\hline & KL1196 & Absent \\
\hline & C. pseudotuberculosis DSM20689 & Absent \\
\hline
\end{tabular}

The results of core genome phylogeny showed that W25 has separated from other $C$. ulcerans genomes and formed a distinct cluster with two other strains, PO100/5 and KL1196 (Fig. 3). The latter two strains are also submitted to the GenBank as C. ulcerans isolates. The remaining $26 C$. ulcerans strains formed two distinct subgroups, which is in agreement with our previous study showing an existence of two lineages within this species (Subedi et al. 2018). The composition of these lineages is highly consistent between the two studies with five additional strains (211, FH2016-1, NCTC 7908, NCTC 7910 and NCTC 8639) grouped in lineage 1 and two additional strains (03-8664 and NCTC 8666) grouped in lineage 2 (Fig. 3) in this study.

Pairwise dDDH values of strain W25 with other $C$. ulcerans isolates and type strains of $C$. belfanti, $C$. diphtheriae and C. pseudotuberculosis, indicate that strain W25 belong to a novel species along with PO100/5 and KL1196 (Table 5). These results are also confirmed by the ANI values. The ANI values between strains W25, PO100/5 and KL1196 were >99\%, consistent with them being the same species but were $<92 \%$ between these strains and C. ulcerans genomes (Supplementary Table 3 ).

An analysis of the genome sequence of strain W25 using PathoBacTyper (Tsai et al. 2017) showed 29\% coverage rate against Corynebacterium variabile strain and Type (Strain) Genome Server (MeierKolthoff and Göker 2019) indicated that this strain belongs to a novel species. Similarly, TrueBac ${ }^{\mathrm{TM}}$ ID cloud system revealed $C$. ulcerans to be the closest species with $90.85 \%$ ANI (90.6\% ANI coverage), $99.72 \%$ similarity between the 16S rRNA, 94.95\% similarity between recA and $99.09 \%$ sequence similarity between $r p l C$ genes of the two strains. These 
Tree scale: 0.1

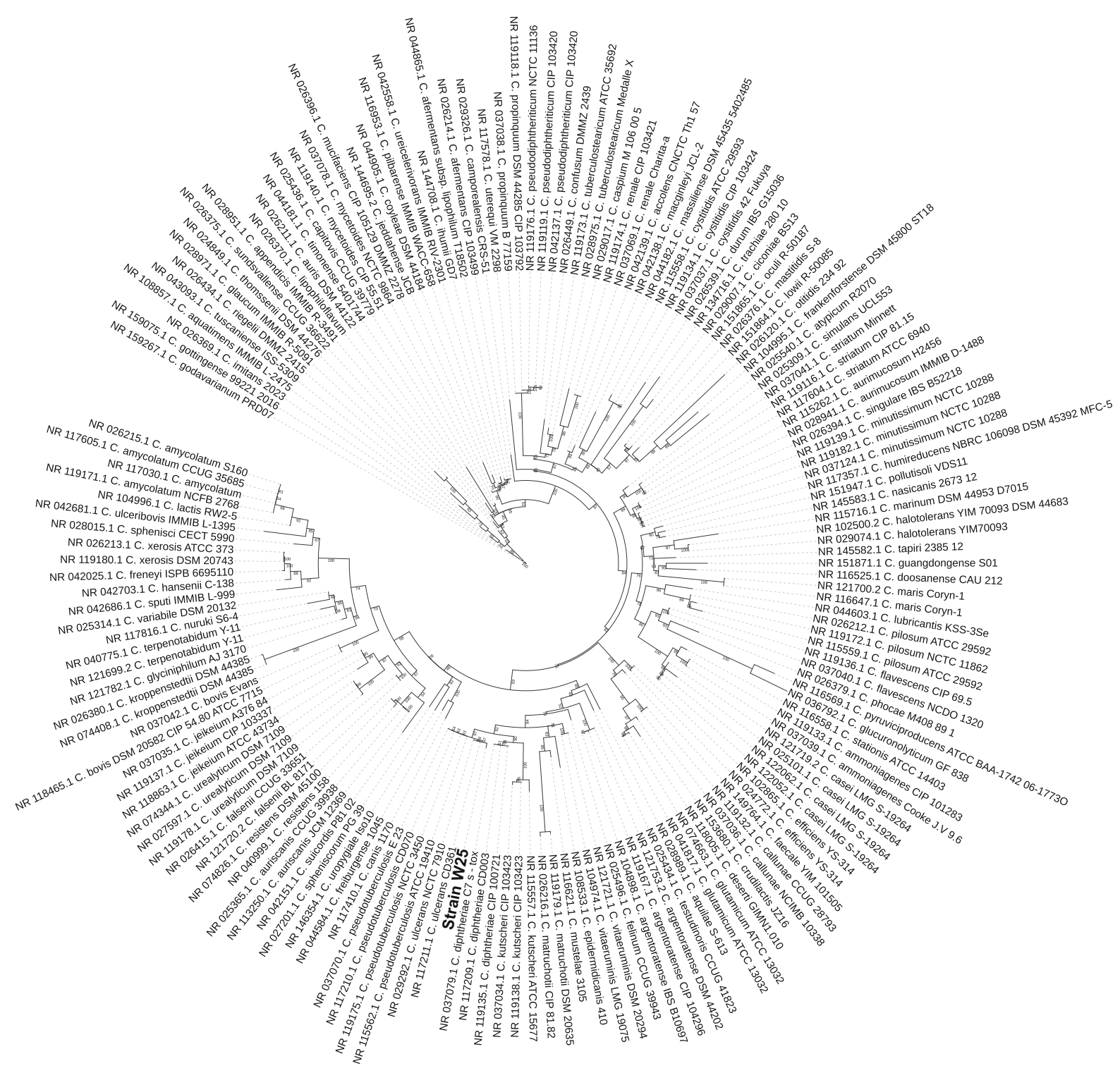

Fig. 2 Maximum likelihood tree from the alignment of 16S rRNA sequences for all Corynebacterium species. The scale bar represents nucleotide substitution per site

results indicate that strains W25 together with PO100/ 5 and KL1196 should be separated from C. ulcerans as a novel species.

A comparative genomic analysis of all C. ulcerans and W25, PO100/5 and KL1196 strains using Roary (Page et al. 2015) with a minimum BLASTP identity of $70 \%$, revealed a pangenome encompassing 4525 genes, of which 1,555 genes belonged to the core genome. The number of genes on individual genomes varied between 2159 and 2529; therefore, $>61 \%$ of the genome is conserved between the two species. Only 30 genes were found to be unique to $C$. ulcerans strains including 23 genes encoding hypothetical proteins (Supplementary Table 4). Homologs of six 


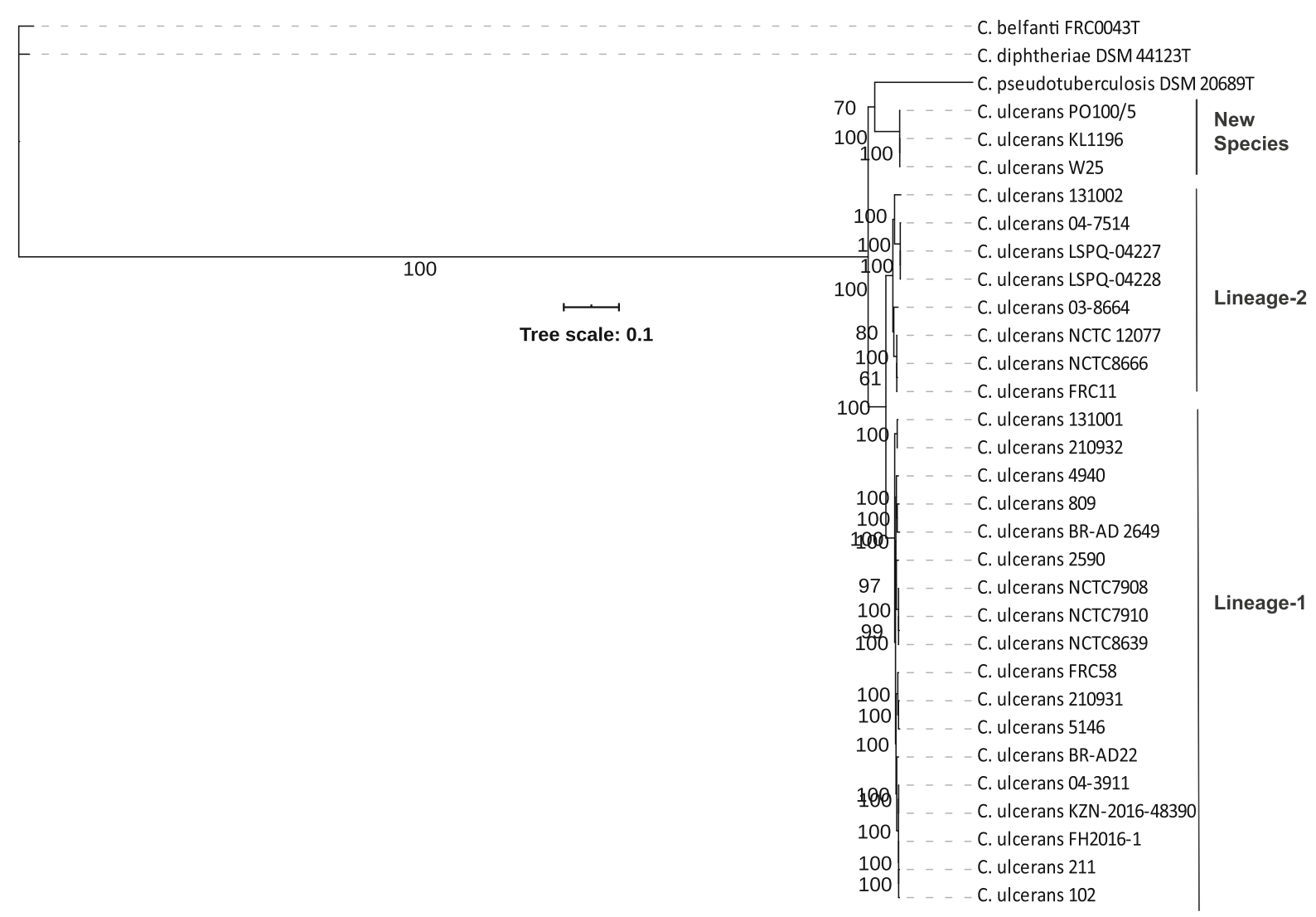

Fig. 3 Maximum likelihood tree from the core genome alignment. The scale bar represents nucleotide substitution per site

of the remaining seven genes encoding aminopeptidase $\mathrm{N}$, cysteine-tRNA ligase, 1,4-dihydroxy-2-naphthoyl-CoA synthase, heat-inducible transcription repressor HrcA, putative fluoride ion transporter $\mathrm{CrcB}$ and putative propionyl-CoA carboxylase beta chain 5 (AccD5) are also present among strains of the novel group. However, RNA polymerase sigma factor YlaC appears to be unique to $C$. ulcerans strains. In contrast, 238 genes were unique to strains W25, PO100/5 and KL1196 (Supplementary Table 5) that were absent among C. ulcerans isolates. Again, 92\% (220 genes) of these genes encode hypothetical/putative proteins. Some of the unique genes encoding $\mathrm{ABC}$ transporter ATP-binding proteins, a UDP-glucose 6-dehydrogenase, glucose-specific EIIA component of phosphotransferase system, proteins involved in hemin transport system (HmuU), vitamin B12 import system (BtuC) and resistance to daunorubicin/doxorubicin (DrrA) have other copies or homologs that are conserved across all the strains and may not cause any functional variation between $C$. ulcerans strains and those belong to the novel group. Similarly, copies of genes encoding sulfate/thiosulfate import ATPbinding protein (CysA) is present among some $C$. ulcerans strains. Eight genes encoding component of ammonia channel (amt), a $b g l$ operon antiterminator (BglG), D-amino acid dehydrogenase (DadA), glucose-specific EIICBA components of phosphotransferase system, oligopeptide transport system permease protein (OppB), a putative peptidase (cp29_00169) and a transcriptional regulatory protein DesR are unique to strains W25, PO100/5 and KL1196 and may be responsible for minor functional variations between these species (Supplementary Table 5).

Expression of the tox gene in strain W25

Diphtheria toxin is the main virulence factor in toxigenic corynebacteria (Sangal and Hoskisson 2014). The tox gene has been amplified in the multiplex PCR reaction (Fig. 1). 
Table $5 \mathrm{dDDH}$ values using recommended formula \#2 between strain W25 and other C. ulcerans strains and type strains of $C$. belfanti, $C$. diphtheriae and C. pseudotuberculosis

\begin{tabular}{|c|c|c|c|c|}
\hline Reference & $\mathrm{DDH}$ & Model C.I. & Distance & $\mathrm{G}+\mathrm{C}$ difference \\
\hline C. diphtheriae DSM $44123^{\mathrm{T}}$ & 22.1 & {$[19.8-24.5 \%]$} & 0.1986 & 0.91 \\
\hline C. belfanti $\mathrm{FRC} 0043^{\mathbf{T}}$ & 22.6 & [20.4-25.1\%] & 0.1935 & 0.81 \\
\hline C. pseudotuberculosis DSM $20689^{\mathbf{T}}$ & 28.5 & {$[26.2-31 \%]$} & 0.1505 & 2.25 \\
\hline NCTC $7910^{\mathbf{T}}$ & 40.9 & {$[38.4-43.5 \%]$} & 0.0964 & 1.12 \\
\hline 809 & 41 & {$[38.5-43.5 \%]$} & 0.0961 & 1.13 \\
\hline BR-AD22 & 40.9 & {$[38.4-43.5 \%]$} & 0.0964 & 1.04 \\
\hline 102 & 40.9 & {$[38.4-43.5 \%]$} & 0.0964 & 1.08 \\
\hline NCTC 12077 & 41 & {$[38.5-43.6 \%]$} & 0.096 & 1.05 \\
\hline FRC58 & 40.9 & {$[38.4-43.5 \%]$} & 0.0964 & 1.13 \\
\hline 210932 & 41 & {$[38.5-43.5 \%]$} & 0.0962 & 1.12 \\
\hline 210931 & 40.7 & [38.3-43.3\%] & 0.097 & 1.13 \\
\hline FRC11 & 41.3 & {$[38.8-43.9 \%]$} & 0.0951 & 1.09 \\
\hline 5146 & 40.9 & {$[38.4-43.5 \%]$} & 0.0964 & 1.13 \\
\hline 131002 & 41.6 & [39.1-44.1\%] & 0.0943 & 1.06 \\
\hline LSPQ-04227 & 41.5 & [39-44\%] & 0.0946 & 1.04 \\
\hline LSPQ-04228 & 41.5 & [39-44\%] & 0.0946 & 1.04 \\
\hline 131001 & 41 & [38.5-43.5\%] & 0.0962 & 1.12 \\
\hline 04-3911 & 41 & {$[38.5-43.5 \%]$} & 0.0963 & 1.11 \\
\hline $03-8664$ & 42.1 & [39.6-44.6\%] & 0.0927 & 0.96 \\
\hline $04-7514$ & 41.2 & {$[38.7-43.7 \%]$} & 0.0956 & 0.97 \\
\hline KZN-2016-48390 & 40.9 & [38.4-43.5\%] & 0.0964 & 1.05 \\
\hline BR-AD 2649 & 41.1 & [38.6-43.6\%] & 0.0958 & 1.17 \\
\hline 2590 & 40.8 & [38.3-43.3\%] & 0.0969 & 1.14 \\
\hline 4940 & 41 & {$[38.5-43.5 \%]$} & 0.0963 & 1.09 \\
\hline 211 & 40.9 & [38.4-43.5\%] & 0.0964 & 1.08 \\
\hline FH2016-1 & 40.9 & {$[38.5-43.5 \%]$} & 0.0963 & 1.08 \\
\hline NCTC 8666 & 41.2 & {$[38.7-43.7 \%]$} & 0.0956 & 1.05 \\
\hline NCTC 7908 & 40.9 & [38.4-43.5\%] & 0.0964 & 1.12 \\
\hline NCTC 8639 & 40.9 & [38.4-43.5\%] & 0.0964 & 1.12 \\
\hline PO100/5 & 98.5 & [97.7-99\%] & 0.0025 & 0.04 \\
\hline KL1196 & 100 & [100-100\%] & 0 & 0 \\
\hline
\end{tabular}

A Western blot using human antiserum against the toxin was negative for strain W25 (Fig. 4A). Diphtheria toxin was detected in extract from strain KL756 during the induction of iron starvation (Fig. 4A). We also performed an Elek test to check the expression of the gene. Elek test is an agar gel immunodiffusion assay where horse diphtheria antitoxin diffuses towards diphtheria toxin produced by toxigenic strains. A precipitation line forms near the bacterial colonies at the zone of equivalence. No precipitation lines were observed for W25, suggesting that this strain is non-toxigenic (Fig. 4B).
A BLAST-search of the protein sequence of the toxin from $C$. diphtheriae NCTC 13129 (DIP0222) showed significant similarity with the protein encoded by the gene cp29_02234 in strain W25 that was annotated to encode a hypothetical protein. A nucleotide sequence alignment of the tox gene including 100 bp upstream and $100 \mathrm{bp}$ downstream regions from strains W25, PO100/5, KL1196, C. diphtheriae NCTC $13129($ DIP0222) and C. ulcerans 0102 (CULC0102_0213) revealed that the gene in strains W25 and KL1196 has a two base (GG) insertion at position 48 (Supplementary Fig. 1), which introduced a frameshift, leading it to be a pseudogene. Therefore, 


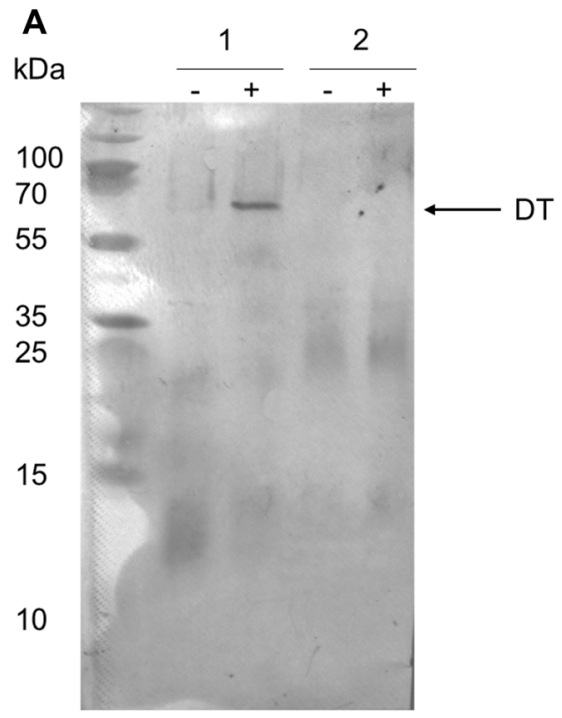

Fig. 4 Detection of diphtheria toxin. A Western blot analysis using DT-specific antiserum and cell extracts from toxigenic $C$. ulcerans KL756 and from strain W25. Bacteria were grown without $(-)$ bipyridyl for control and with $(+)$ this iron chelator to induce iron starvation and induction of tox transcription. $2 \mu \mathrm{g}$ of protein extract were added per lane. Human serum collected 1 year after primary and two booster vaccinations (second serum) was used as primary antibody. 1: KL756 $\left(\operatorname{tox}^{+}\right), 2$ : W25. The

W25 strain is an NTTB strain, which is consistent with nucleotide sequence being amplified in a PCR reaction and Elek test being negative.

To further test the toxin expression, RNA hybridization experiments were carried out using tox ${ }^{+}$C. ulcerans strain KL756 as the positive control and tox ${ }^{-}$strain 809 as the negative control. A presence of $16 \mathrm{~S} r R N A$ gene expression was confirmed in all three strains (Fig. 5A) whereas transcript for tox gene was only detected for strain KL756 when iron starvation was induced by bipyridyl (Fig. 5B). These results confirmed that W25 is an NTTB strain.

Pilus genes clusters in strain W25

Similar to C. ulcerans strains, two spaBC and spaDEF type pilus gene clusters have been identified in strain W25 (Subedi et al. 2018; Trost et al. 2011). The genes encoding sortase $\mathrm{A}$ and $\mathrm{SpaB}$ fimbrial subunit are present in strain W25; however, the C-terminal region of the SpaC fimbrial subunit is truncated and the corresponding gene is annotated as two smaller genes (Fig. 6). Therefore, SpaBC type pili in this strain may not be functional.
B
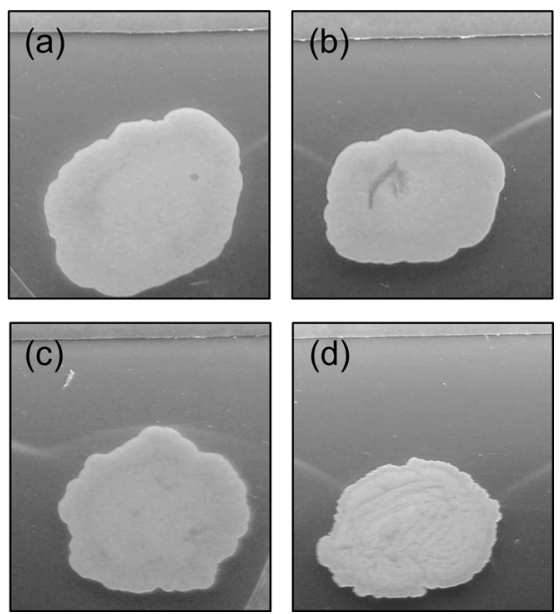

arrow indicates DT with an apparent molecular mass of $62 \mathrm{kDa}$. B Elek test with purified antitoxin. (a): C. ulcerans 809 (tox ${ }^{-}$); (b): C. ulcerans BR-AD22 (tox ${ }^{-}$); (c): C. ulcerans KL756 $\left(\right.$ tox $^{+}$); (d): C. ulcerans W25. As a control C. diphtheriae strains NCTC 10648 (positive control) and NCTC 10356 (negative control) were used. After an incubation of $24 \mathrm{~h}$, precipitation lines for the toxigenic C. ulcerans strain KL756 were detected

Similarly, spaD, srtC and spaF genes are also truncated and annotated as two smaller genes, respectively, and SpaE is missing approximately 65 amino acid residues at the $\mathrm{N}$-terminal region. A small gene encoding hypothetical protein is also present between $s p a E$ and $s p a F$ (Fig. 6). Therefore, SpaDEF type pili may also be absent in strain W25.

\section{Other virulence genes in strain W25}

When searched for other corynebacterial virulence genes, strain W25 was found to possess most of the other virulence genes present in $C$. ulcerans strains including cpp (corynebacterial protease), pld (phospholipase D), nanH (neuraminidase, sialidase), vspl and $v s p 2$ (trypsin-like serine protease) and $c w l H$ (hydrolase; cell wall peptidase; Table 6). A gene annotated as ripA (peptidoglycan endopeptidase) show $91 \%$ identities with the rpfI gene in C. ulcerans with a deletion of 34 amino acids from position 299 to 332. However, two virulence genes $r b p$ and $t s p A$ were absent in strain W25.

Strain W25 also possessed several virulence genes identified in C. pseudotuberculosis strain FRC41 


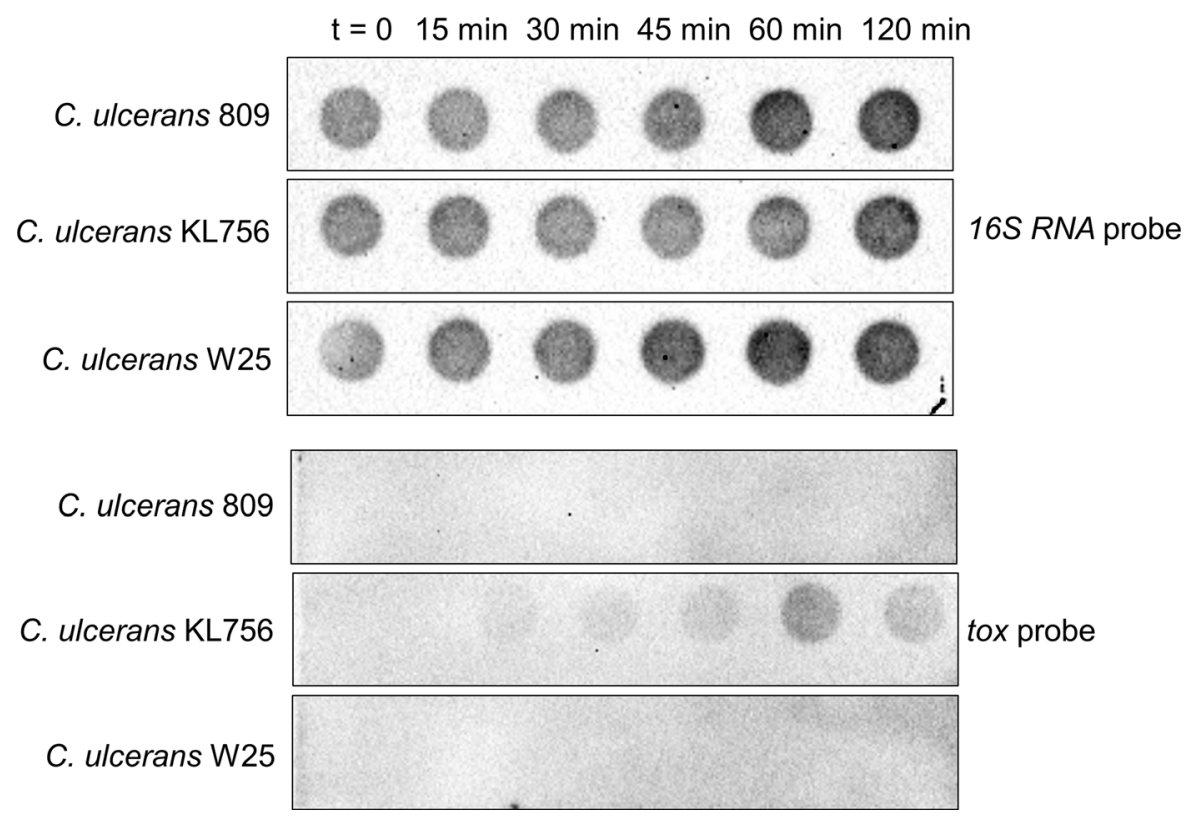

Fig. 5 Transcription of the tox gene. RNA hybridization of $C$. ulcerans strains (tox ${ }^{-}: 809$; tox $^{+}:$KL756) and W25 at six different time points $(\mathrm{t}=0$ : before induction with bipyridyl; 15 ,

spaBC gene cluster (cp29_00127 - cp29_00130)

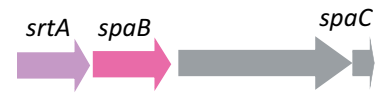

\section{spaDEF gene cluster (cp29_00167-cp29_00175)}

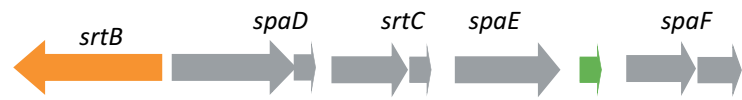

Fig. 6 spa gene clusters in strain W25. The schematic is not to scale. The direction of arrows indicate the orientation of the coding sequence

(Trost et al. 2010). These include cpfrc_00397 (secretion-associated serine protease), dtsRl (acylCoA carboxylase subunit beta), dtsR2 (acyl-CoA carboxylase subunit beta), accD3 (propionyl-CoA carboxylase beta chain 2), cpfrc_00536 (secreted SGNH-hydrolase), nrpS1 (nonribosomal peptide synthetase 1) as well as $r p f A$ and $r p f B$ (resuscitationpromoting factors). However, two genes nor (nitric oxide reductase) and cpfrc_00562 (secreted trypsinlike serine protease) were found to be absent.
30, 45, 60 and 120 min post induction). A 16SrRNA probe was used as a control. RNA hybridization experiments were carried out in three independent biological replicates

\section{Discussion}

C. ulcerans and C. pseudotuberculosis are pathogens adapted to canine and ovine hosts, respectively but can cause zoonotic infections in humans (Bregenzer et al. 1997; Hacker et al. 2016; Peel et al. 1997). In this study, we have characterised an isolate W25, which has been identified as an atypical $C$. ulcerans based in MALDI-TOF analysis, multiplex PCR using $16 \mathrm{~S}$ rRNA, rрoB and tox genes (Fig. 1) and other biochemical characteristics (Table 3).

Genome-based matrices have been extensively used to define novel bacterial species (Nouioui et al. 2018, 2019; Sangal et al. 2016, 2018). The dDDH and ANI cut-off values for defining new species are $70 \%$ and 95\%, respectively (Auch et al. 2010b; Konstantinidis and Tiedje 2005, 2007). The genome sequences of strains W25 showed $>98 \% \mathrm{dDDH}$ and $>99 \%$ ANI values against the genome sequences of strains PO100/5 and KL1196, clearly indicating that these strains belong to the same species. These strains are phylogenetically closely related to other pathogenic corynebacteria, $C$. diphtheriae, $C$. pseudotuberculosis and C. ulcerans (Figs. 2 and 3). However, dDDH and ANI values were below the species cut-off between W25 and the type strains of these species (Table 5; 
Table 6 Other virulence genes in strain W25

\begin{tabular}{lll}
\hline Gene & Gene in W25 & Protein \\
\hline pld & cp29_02424 & Phospholipase D \\
nanH & cp29_01937 & Neuraminidase (sialidase) \\
vsp1 & cp29_01856 & Trypsin-like serine protease \\
ripA & cp29_01144 & Peptidoglycan endopeptidase \\
cwlH & cp29_00749 & Hydrolase (cell wall peptidase) \\
vsp2 & cp29_00148 & Protease \\
cpp & cp29_00135 & Protease (endo-beta-N-acetylglucosaminidase F2) \\
cpfrc_00397 & cp29_01926 & Type VII secretion-associated serine protease mycosin \\
dtsR2 & cp29_01827 & AccD5-3 acyl-CoA carboxylase subunit beta \\
dtsR1 & cp29_01826 & AccD5-2 propionyl-CoA carboxylase beta chain 2 \\
accD3 & cp29_00074 & AccD5-1 acyl-CoA carboxylase subunit beta \\
cpfrc_00536 & cp29_01777 & Hydrolase \\
$n r p S 1$ & $c p 29 \_01752$ & Non-ribosomal peptide synthetase \\
$r f p A$ & cp29_01715 & Resuscitation-promoting factor \\
$r f p B$ & cp29_01628 & Resuscitation-promoting factor \\
\hline
\end{tabular}

Supplementary Table 3), suggesting that this strain belong to a novel Corynebacterium species along with two other strains PO100/5 and KL1196. These results are also confirmed by other bacterial identification platforms including PathoBacTyper (Tsai et al. 2017), TrueBac $^{\mathrm{TM}}$ ID cloud system (www.truebacid.com) and Type (Strain) Genome Server (Meier-Kolthoff and Göker 2019). W25 was isolated from a case of necrotizing lymphadenitis in a wild boar (Busch et al. 2019), PO100/5 from a pig and KL1196 from a roe deer. Therefore, this species also appears to be prevalent among animals.

One of the key biochemical difference separating strain W25 from $C$. ulcerans was inability of the former to ferment starch (Table 3). We identified protein sequences for pullulanase type I and 1,4-alpha amylase enzymes that are involved in starch metabolism are absent in strain W25 as well as PO100/5 and KL1196 (Table 4). The genes encoding these enzymes are inactive (pseudogenes) due to frameshift mutations among these isolates. While both the genes are present in $C$. ulcerans, the gene encoding 4-alpha amylase enzyme is absent in $C$. pseudotuberculosis (Table 4). In general, C. pseudotuberculosis strains do not ferment starch (Dorella et al. 2006). Therefore, both the enzymes seem to be important for starch metabolism and an absence of any of these may compromise the ability to ferment starch.

Interestingly, the genome of strain W25 has been annotated to carry the tox gene, encoding a diphtheria- like toxin but the protein was not detectable in the Western blot using human antiserum against the toxin (Fig. 4A) or in the Elek test (Fig. 4B). Furthermore, no transcript of the tox gene was observed in the RNA hybridization experiment (Fig. 5A, B). The gene has a two base (GG) insertion at position 48 which has introduced the frameshift (Supplementary Fig. 1).

Two pilus gene clusters ( $s p a B C$ and $s p a D E F$ ) have been identified in strain W25; however, both of them show potential loss of the gene functions (Fig. 6). A $s p a B C$ cluster is also present in C. ulcerans, which lacks the spaA gene encoding a major pilin subunit (Subedi et al. 2018; Trost et al. 2011). SpaABC type pili are known to interact with pharyngeal epithelial cells (Mandlik et al. 2007; Reardon-Robinson and Ton-That 2014) and homodimeric or heterodimeric $\mathrm{SpaB} / \mathrm{SpaC}$ proteins were suggested to facilitate this interaction (Trost et al. 2011). However, spaC gene encoding the tip protein is also truncated in strain W25. Similarly, multiple genes of the the spaDEF cluster are truncated in strain W25 (Fig. 6). These pili in $C$. diphtheriae are characterised to interact with laryngeal epithelial cells (Mandlik et al. 2007; Reardon-Robinson and Ton-That 2014). The spaDEF cluster is characterised of five genes, $s p a D, s p a E$ and $s p a F$ encoding the major pilin subunit, minor subunit and the tip protein, respectively and two sortasesencoding genes, $s r t B$ and $s r t C$, responsible assembly of the pilus (Mandlik et al. 2007; Reardon-Robinson and Ton-That 2014). Therefore, it is possible that the 
ability of this strain to interact with the pharyngeal or laryngeal epithelial cells is compromised.

However, the strain W25 possesses a number of virulence genes present among $C$. ulcerans and $C$. pseudotuberculosis strains, which may enable it to cause severe invasive infections (Table 6). For example, phospholipase $\mathrm{D}$ is a well-characterised virulenceassociated protein responsible for significant macrophage death (McKean et al. 2007). Similarly, protease and hydrolases activities of other proteins have been found to contribute to the virulence properties in pathogenic corynebacteria (Trost et al. 2010, 2011).

\section{Conclusions}

Isolate W25 is biochemically similar to C. ulcerans strains that can produce $\mathrm{H}_{2} \mathrm{~S}$ on Tinsdale medium, is positive for reverse CAMP reaction and hydrolase activity, and is able to utilise glucose as a carbon source (Table 3 ). This strain was previously defined as atypical $C$. ulcerans but belong to a novel species including two strains, PO100/5 and KL1196, which were independently isolated from animals. The isolate is likely an NTTB strain with compromised abilities to adhere to pharyngeal and laryngeal epithelial cells due to loss of the multiple genes in $s p a B C$ and $s p a D E F$ pilus gene clusters. However, a number of corynebacterial virulence genes are present, which may enable the strain to cause severe invasive infections in animals and zoonotic infections in humans.

Author contribution $\mathrm{AB}$ and VS conceived the idea and designed the study. JM, VM and WG performed the phenotypic characterisation of corynebacterial strains. LM and VS analysed the genomic data. JM, AB and VS drafted the manuscript. All authors read and approved the manuscript.

\section{Compliance with ethical standards}

Conflict of interest The author declare that they have no conflict of interest.

Human and animal participants This study does not involve any human participants or animal experiments.

Open Access This article is licensed under a Creative Commons Attribution 4.0 International License, which permits use, sharing, adaptation, distribution and reproduction in any medium or format, as long as you give appropriate credit to the original author(s) and the source, provide a link to the
Creative Commons licence, and indicate if changes were made. The images or other third party material in this article are included in the article's Creative Commons licence, unless indicated otherwise in a credit line to the material. If material is not included in the article's Creative Commons licence and your intended use is not permitted by statutory regulation or exceeds the permitted use, you will need to obtain permission directly from the copyright holder. To view a copy of this licence, visit http://creativecommons.org/licenses/by/4.0/.

\section{References}

Abe S, Takayama K, Kinoshita S (1967) Taxonomical studies on glutamic acid producing bacteria. J Gen Microbiol 13:279-301

Alibi S, Ferjani A, Gaillot O, Marzouk M, Courcol R, Boukadida J (2015) Identification of clinically relevant Corynebacterium strains by Api Coryne, MALDI-TOF-mass spectrometry and molecular approaches. Pathol Biol (Paris) 63:153-157. https://doi.org/10.1016/j.patbio.2015. 07.007

Altschul SF, Madden TL, Schaffer AA, Zhang J, Zhang Z, Miller W, Lipman DJ (1997) Gapped BLAST and PSIBLAST: a new generation of protein database search programs. Nucleic Acids Res 25:3389-3402

Auch AF, Klenk HP, Göker M (2010a) Standard operating procedure for calculating genome-to-genome distances based on high-scoring segment pairs. Stand Genomic Sci 2:142-148. https://doi.org/10.4056/sigs.541628

Auch AF, von Jan M, Klenk HP, Göker M (2010b) Digital DNA-DNA hybridization for microbial species delineation by means of genome-to-genome sequence comparison. Stand Genomic Sci 2:117-134. https://doi.org/10. 4056/sigs.531120

Bernard KA, Funke G (2015) Corynebacterium. In: Whitman WB et al. (ed) Bergey's manual of systematics of archaea and bacteria. Wiley, London, pp 1-70 https://doi.org/10. 1002/9781118960608.gbm00026

Bosi E et al (2015) MeDuSa: a multi-draft based scaffolder. Bioinformatics 31:2443-2451. https://doi.org/10.1093/ bioinformatics/btv 171

Bregenzer T, Frei R, Ohnacker H, Zimmerli W (1997) Corynebacterium pseudotuberculosis infection in a butcher. Clin Microbiol Infect 3:696-698. https://doi.org/10.1111/j. 1469-0691.1997.tb00482.x

Broadway MM et al (2013) Pilus gene pool variation and the virulence of Corynebacterium diphtheriae clinical isolates during infection of a nematode. $\mathrm{J}$ Bacteriol 195:3774-3783. https://doi.org/10.1128/JB.00500-13

Busch A, Moller J, Burkovski A, Hotzel H (2019) Genome sequence of a pathogenic Corynebacterium ulcerans strain isolated from a wild boar with necrotizing lymphadenitis. BMC Res Notes 12:692. https://doi.org/10.1186/s13104019-4704-3

Camacho C, Coulouris G, Avagyan V, Ma N, Papadopoulos J, Bealer K, Madden TL (2009) BLAST + : architecture and applications. BMC Bioinform 10:421. https://doi.org/10. 1186/1471-2105-10-421 
Dias AA et al (2010) Corynebacterium ulcerans isolated from an asymptomatic dog kept in an animal shelter in the metropolitan area of Rio de Janeiro, Brazil. Vector Borne Zoonotic Dis 10:743-748. https://doi.org/10.1089/vbz. 2009.0132

Dias AA et al (2011) Strain-dependent arthritogenic potential of the zoonotic pathogen Corynebacterium ulcerans. Vet Microbiol 153:323-331. https://doi.org/10.1016/j.vetmic. 2011.06.007

Dorella FA, Pacheco LG, Oliveira SC, Miyoshi A, Azevedo V (2006) Corynebacterium pseudotuberculosis: microbiology, biochemical properties, pathogenesis and molecular studies of virulence. Vet Res 37:201-218

Edgar RC (2004) MUSCLE: multiple sequence alignment with high accuracy and high throughput. Nucleic Acids Res 32:1792-1797. https://doi.org/10.1093/nar/gkh340

Efstratiou A, George RC (1999) Laboratory guidelines for the diagnosis of infections caused by Corynebacterium diphtheriae and C. ulcerans. Commun Dis Public Health 2:250-257

Eisenberg T, Kutzer P, Peters M, Sing A, Contzen M, Rau J (2014) Nontoxigenic tox-bearing Corynebacterium ulcerans infection among game animals, Germany. Emerg Infect Dis 20:448-452. https://doi.org/10.3201/eid2003. 130423

Fuursted K, Soes LM, Crewe BT, Stegger M, Andersen PS, Christensen JJ (2015) Non-toxigenic tox gene-bearing Corynebacterium ulcerans in a traumatic ulcer from a human case and his asymptomatic dog. Microbes Infect 17:717-719. https://doi.org/10.1016/j.micinf.2015.07.004

Grosse-Kock S et al (2017) Genomic analysis of endemic clones of toxigenic and non-toxigenic Corynebacterium diphtheriae in Belarus during and after the major epidemic in 1990s. BMC Genom 18:873. https://doi.org/10.1186/ s12864-017-4276-3

Ha SM et al (2019) Application of the whole genome-based bacterial identification system, TrueBac ID, using clinical isolates that were not identified with three matrix-assisted laser desorption/ionization time-of-flight mass spectrometry (MALDI-TOF MS) systems. Ann Lab Med 39:530-536

Hacker E, Antunes CA, Mattos-Guaraldi AL, Burkovski A, Tauch A (2016) Corynebacterium ulcerans, an emerging human pathogen. Future Microbiol 11:1191-1208. https:// doi.org/10.2217/fmb-2016-0085

Jain C, Rodriguez RL, Phillippy AM, Konstantinidis KT, Aluru S (2018) High throughput ANI analysis of 90 K prokaryotic genomes reveals clear species boundaries. Nat Commun 9:5114. https://doi.org/10.1038/s41467-018-07641-9

Konrad R, Hormansdorfer S, Sing A (2015) Possible human-tohuman transmission of toxigenic Corynebacterium ulcerans. Clin Microbiol Infect 21:768-771. https://doi.org/10. 1016/j.cmi.2015.05.021

Konstantinidis KT, Tiedje JM (2005) Genomic insights that advance the species definition for prokaryotes. Proc Natl Acad Sci U S A 102:2567-2572. https://doi.org/10.1073/ pnas.0409727102

Konstantinidis KT, Tiedje JM (2007) Prokaryotic taxonomy and phylogeny in the genomic era: advancements and challenges ahead. Curr Opin Microbiol 10:504-509. https:// doi.org/10.1016/j.mib.2007.08.006
Lagesen K, Hallin P, Rodland EA, Staerfeldt HH, Rognes T, Ussery DW (2007) RNAmmer: consistent and rapid annotation of ribosomal RNA genes. Nucleic Acids Res 35:3100-3108. https://doi.org/10.1093/nar/gkm160

Letunic I, Bork P (2016) Interactive tree of life (iTOL) v3: an online tool for the display and annotation of phylogenetic and other trees. Nucleic Acids Res 44:W242-W245. https://doi.org/10.1093/nar/gkw290

Mandlik A, Swierczynski A, Das A, Ton-That H (2007) Corynebacterium diphtheriae employs specific minor pilins to target human pharyngeal epithelial cells. Mol Microbiol 64:111-124. https://doi.org/10.1111/j.1365-2958.2007. 05630.x

Mattos-Guaraldi AL et al (2008) First detection of Corynebacterium ulcerans producing a diphtheria-like toxin in a case of human with pulmonary infection in the Rio de Janeiro metropolitan area, Brazil. Mem Inst Oswaldo Cruz 103:396-400. https://doi.org/10.1590/s007402762008000400014

Mattos-Guaraldi AL, Hirata R Jr, Azevedo VA (2014) Corynebacterium diphtheriae, Corynebacterium ulcerans and Corynebacterium pseudotuberculosis: general aspects. In: Burkovski A (ed) Corynebacterium diphtheriae and related toxigenic species. Springer, Heidelberg, pp 15-37

Mazurova IK, Melnikov VG, Kombarova SJ, Borisova OY, Zhilina NY (1998) Manual for diphtheria laboratory diagnosis. Russ Fed State Comm Sanit Epidemiol Surv 7

McKean SC, Davies JK, Moore RJ (2007) Expression of phospholipase $\mathrm{D}$, the major virulence factor of $\mathrm{Co}$ rynebacterium pseudotuberculosis, is regulated by multiple environmental factors and plays a role in macrophage death. Microbiology 153:2203-2211. https://doi.org/10. 1099/mic.0.2007/005926-0

Meier-Kolthoff JP, Göker M (2019) TYGS is an automated high-throughput platform for state-of-the-art genomebased taxonomy. Nat Commun 10:2182. https://doi.org/10. 1038/s41467-019-10210-3

Meinel DM, Margos G, Konrad R, Krebs S, Blum H, Sing A (2014) Next generation sequencing analysis of nine Corynebacterium ulcerans isolates reveals zoonotic transmission and a novel putative diphtheria toxin-encoding pathogenicity island. Genome Med 6:113. https://doi.org/ 10.1186/s13073-014-0113-3

Möller J et al (2019) Proteomics of diphtheria toxoid vaccines reveals multiple proteins that are immunogenic and may contribute to protection of humans against Corynebacterium diphtheriae. Vaccine 37:3061-3070. https://doi. org/10.1016/j.vaccine.2019.04.059

Moreira LO et al (2003) Effects of iron limitation on adherence and cell surface carbohydrates of Corynebacterium diphtheriae strains. Appl Environ Microbiol 69:5907-5913. https://doi.org/10.1128/aem.69.10.5907-5913.2003

Nguyen LT, Schmidt HA, von Haeseler A, Minh BQ (2015) IQTREE: a fast and effective stochastic algorithm for estimating maximum-likelihood phylogenies. Mol Biol Evol 32:268-274. https://doi.org/10.1093/molbev/msu300

Nouioui I, Ghodhbane-Gtari F, Rhode M, Sangal V, Klenk HP, Gtari M (2018) Frankia irregularis sp nov., an actinobacterium unable to nodulate its original host, Casuarina equisetifolia, but effectively nodulates members of the 
actinorhizal Rhamnales. Int J Syst Evol Microbiol 68:2883-2890. https://doi.org/10.1099/ijsem.0.002914

Nouioui I et al (2019) Mycolicibacterium stellerae sp. nov., a rapidly growing scotochromogenic strain isolated from Stellera chamaejasme. Int J Syst Evol Microbiol 69:3465-3471. https://doi.org/10.1099/ijsem.0.003644

Ott L, Holler M, Rheinlaender J, Schaffer TE, Hensel M, Burkovski A (2010) Strain-specific differences in pili formation and the interaction of Corynebacterium diphtheriae with host cells. BMC Microbiol 10:257. https://doi.org/10. 1186/1471-2180-10-257

Page AJ et al (2015) Roary: rapid large-scale prokaryote pan genome analysis. Bioinformatics 31:3691-3693. https:// doi.org/10.1093/bioinformatics/btv421

Peel MM, Palmer GG, Stacpoole AM, Kerr TG (1997) Human lymphadenitis due to Corynebacterium pseudotuberculosis: report of ten cases from Australia and review. Clin Infect Dis 24:185-191. https://doi.org/10.1093/clinids/24. 2.185

Reardon-Robinson ME, Ton-That H (2014) Assembly and function of Corynebacterium diphtheriae pili. In: Burkovski A (ed) Corynebacterium diphtheriae and related toxigenic species. Springer, Heidelberg, pp 123-141. https://doi.org/10.1007/978-94-007-7624-1

Sangal V, Hoskisson PA (2014) Corynephages: infections of the infectors. In: Burkovski A (ed) Corynebacterium diphtheriae and related toxigenic species. Springer, Heidelberg, pp 67-82. https://doi.org/10.1007/978-94-007-7624-1

Sangal V et al (2014) Diphtheria-like disease caused by toxigenic Corynebacterium ulcerans strain. Emerg Infect Dis 20:1257-1258. https://doi.org/10.3201/eid2007.140216

Sangal V, Blom J, Sutcliffe IC, von Hunolstein C, Burkovski A, Hoskisson PA (2015) Adherence and invasive properties of Corynebacterium diphtheriae strains correlates with the predicted membrane-associated and secreted proteome. BMC Genom 16:765. https://doi.org/10.1186/s12864-0151980-8

Sangal V, Goodfellow M, Jones AL, Schwalbe EC, Blom J, Hoskisson PA, Sutcliffe IC (2016) Next-generation systematics: an innovative approach to resolve the structure of complex prokaryotic taxa. Sci Rep 6:38392. https://doi. org/10.1038/srep38392

Sangal V, Goodfellow M, Blom J, Tan GYA, Klenk HP, Sutcliffe IC (2018) Revisiting the taxonomic status of the biomedically and industrially important genus Amycolatopsis, using a phylogenomic approach. Front Microbiol 9:2281. https://doi.org/10.3389/fmicb.2018.02281

Seemann T (2014) Prokka: rapid prokaryotic genome annotation. Bioinformatics 30:2068-2069. https://doi.org/10. 1093/bioinformatics/btu153

Sekizuka T et al (2012) Corynebacterium ulcerans 0102 carries the gene encoding diphtheria toxin on a prophage different from the $C$. diphtheriae NCTC 13129 prophage. BMC Microbiol 12:72. https://doi.org/10.1186/1471-2180-12-72

Sievers F et al (2011) Fast, scalable generation of high-quality protein multiple sequence alignments using Clustal Omega. Mol Syst Biol 7:539

Subedi R et al (2018) Genomic analyses reveal two distinct lineages of Corynebacterium ulcerans strains. New Microbes New Infect 25:7-13. https://doi.org/10.1016/j. nmni.2018.05.005

Tange O (2011) GNU parallel: the command-line power tool login: the USENIX Magazine, 42-47

Torres Lde F et al (2013) Multiplex polymerase chain reaction to identify and determine the toxigenicity of Corynebacterium spp with zoonotic potential and an overview of human and animal infections. Mem Inst Oswaldo Cruz. https://doi.org/10.1590/S0074-02762013000300003

Trost E et al (2010) The complete genome sequence of Corynebacterium pseudotuberculosis FRC41 isolated from a 12-year-old girl with necrotizing lymphadenitis reveals insights into gene-regulatory networks contributing to virulence. BMC Genom 11:728. https://doi.org/10.1186/ 1471-2164-11-728

Trost E et al (2011) Comparative analysis of two complete Corynebacterium ulcerans genomes and detection of candidate virulence factors. BMC Genom 12:383. https://doi. org/10.1186/1471-2164-12-383

Tsai MH, Liu YY, Soo VW (2017) PathoBacTyper: a web server for pathogenic bacteria identification and molecular genotyping. Front Microbiol 8:1474. https://doi.org/10. 3389/fmicb.2017.01474

Wagner KS et al (2011) Screening for Corynebacterium diphtheriae and Corynebacterium ulcerans in patients with upper respiratory tract infections 2007-2008: a multicentre European study. Clin Microbiol Infect 17:519-525. https:// doi.org/10.1111/j.1469-0691.2010.03269.x

Weerasekera D, Moller J, Kraner ME, Azevedo Antunes C, Mattos-Guaraldi AL, Burkovski A (2019) Beyond diphtheria toxin: cytotoxic proteins of Corynebacterium ulcerans and Corynebacterium diphtheriae. Microbiology 165:876-890. https://doi.org/10.1099/mic.0.000820

Zakikhany K, Neal S, Efstratiou A (2014) Emergence and molecular characterisation of non-toxigenic tox genebearing Corynebacterium diphtheriae biovar mitis in the United Kingdom, 2003-2012. Euro Surveill. https://doi. org/10.2807/1560-7917.es2014.19.22.20819

Publisher's Note Springer Nature remains neutral with regard to jurisdictional claims in published maps and institutional affiliations. 